\title{
The Danish Multidisciplinary Hip Fracture Registry 13-Year Results from a Population-Based Cohort of Hip Fracture Patients
}

This article was published in the following Dove Press journal:

Clinical Epidemiology

\author{
Pia Kjær Kristensen (D) ${ }^{1,2}$ \\ Niels Dieter Röck (iD) ${ }^{3}$ \\ Helle Collatz Christensen ${ }^{4}$ \\ Alma Becic Pedersen (D) \\ 'Department of Clinical Epidemiology, \\ Aarhus University Hospital, Aarhus, \\ Denmark; ${ }^{2}$ Department of Orthopaedic \\ Surgery, Horsens Regional Hospital, \\ Horsens, Denmark; ${ }^{3}$ Department of \\ Orthopaedic Surgery, Odense University \\ Hospital, Odense, Denmark; ${ }^{4}$ Danish \\ Clinical Quality Program (RKKP) National \\ Clinical Registries, Frederiksberg Hospital, \\ Frederiksberg, Denmark
}

\begin{abstract}
Aim of the Registry: The aim of the Danish Multidisciplinary Hip Fracture Registry (DMHFR) is to collect data on processes of treatment, nursing care and rehabilitation as well as outcomes for patients with hip fracture in Denmark, and thereby monitor and improve the quality.
\end{abstract}

Study Population: Hip fracture patients at age 65 or older that have undergone surgery with arthroplasty or internal fixation since 2004.

Main Variables: DMHFR collects quality indicators and descriptive variables. Quality indicators include eight process performance measures within treatment, nursing care and rehabilitation, reflecting recommendations from the national clinical guideline for hip fracture patients, and three outcome measures including survival within 30-days, unplanned acute readmission within 30 days and reoperation within 2 years. Descriptive variables include a number of patient- and surgery-related characteristics. All data are collected prospectively.

Results: By the end of 2018, the DMHFR included 86,438 hip fracture patients. Since 2006, all hospital departments in Denmark, treating patients with hip fracture, have reported improvement in quality of care and improvement in survival, and reoperation over time as well as high completeness of variables registration.

Conclusion: The DMHFR is a well-established nationwide clinical registry, which plays a key role for monitoring and improving hip fracture care in Denmark. The registry can further be linked to a range of other nationwide registries in order to answer a number of relevant clinical research questions.

Keywords: hip fracture, quality indicators, registry, mortality, reoperation, readmission

\section{Introduction}

The number of patients hospitalized due to hip fracture has been reported to be 620,000 in the European Union in 2010 and 210,000 per year between 2008 and 2011 in the United States. ${ }^{1,2}$ It is the dominant cause of trauma-related mortality in people above 65 years, and among the survivors, 50\% never reached their previous functional level. ${ }^{3-6}$ Moreover, hip fracture is related to considerable healthcare costs. $^{7-9}$ Previous research has indicated variation in outcome after hip fracture and only a slight improvement in survival over time. ${ }^{10-12}$ Western healthcare systems have therefore developed clinical guidelines for hip fracture care and countries including Sweden, Norway, Finland, Denmark and the United Kingdom have initiated continuous monitoring of the quality of care after hip fracture. ${ }^{13,14}$
Correspondence: Pia Kjær Kristensen Department of Clinical Epidemiology, Aarhus University Hospital, Olof Palmes Allé 43-45, Aarhus DK-8200, Denmark Tel +4587168063

Fax +4587167215

Email pkkr@clin.au.dk 
The Danish Multidisciplinary Hip Fracture Registry (DMHFR) was initiated in 2003 as part of a national quality improvement project called the Danish National Indicator Project. ${ }^{15}$ The aim of the DMHFR is to monitor and support implementation of evidence-based clinical guidelines for hip fracture care and ultimately improve the quality and outcome of hip fracture patients. The registry has a multidisciplinary steering committee appointed by the Danish regions, the Danish Orthopedic Society, the Danish Geriatric Society, the Danish Nurses Organization and the Danish Society for Physiotherapists. ${ }^{16}$ The steering committee chose quality of care indicators, including process performance measures and outcome, as well as descriptive prognostic variables, which reflect current evidence-based approaches within multidisciplinary hip fracture care and their feasibility in clinical practice. The DMHFR is part of the umbrella organization Danish Clinical Registries, and the results are publically available in an annual report in aggregated form. ${ }^{16}$

\section{Aim}

The present paper aims to describe the organization and content of the DMHFR, to present results regarding demographics and quality indicators during the period 2006-2018 and to illustrate the potential of the DMHFR for clinical epidemiological research.

\section{Materials and Methods}

\section{Setting}

Denmark is a country with 5.7 million inhabitants with free access to medical care and a longstanding tradition for health-care registries. ${ }^{17}$ All patients with hip fracture are admitted to the nearest public hospital treating acute patients. All inhabitants have a unique civil registration number, which is used in all healthcare contacts and allows unambiguous linkage between the healthcare registries. ${ }^{18}$

\section{Data Collection Procedures}

Reporting to the DMHFR is mandatory by law for all hospitals from 2006. ${ }^{19}$ During the period from 2006 to 2018 all hospitals treating hip fracture patients were reporting to the registry. Data are prospectively collected on an individual-level by healthcare professionals involved in treatment of hip fracture patients from the time of hospital admission to discharge, covering different aspects of the clinical pathway. Detailed data definitions are developed prior to data collection. Fulfillment of the process performance measures are registered by the staff members prospectively as part of the clinical routine and are reported monthly to the registry. From 2004 to March 2010 the registry was a web-based standalone database, as data were collected using an independent web-based interface. From March 2010 and forward, the registry uses routine collected data retrieved directly from the Danish National Patient Registry to avoid double registration by clinicians. ${ }^{20}$

\section{Study Population}

The DMHFR includes patients' age $\geq 65$ admitted acutely with a femoral neck, pertrochanteric (intertrochanterica femoris or trochanterica femoris) or subtrochanteric fracture (Figure 1) and treated surgically with osteosynthesis or total/hemi arthroplasty.

\section{Main Variables \\ Quality Indicators}

A documentary report, which reflects current evidence within multidisciplinary hip fracture care, is developed. On the basis of this evidence-based approach as well as feasibility in clinical practice, quality of care indicators are developed. The quality indicators in the DMHFR include process performance measures and outcome measures. Eight process performance measures are currently available and include timing of preoperative optimization, surgery

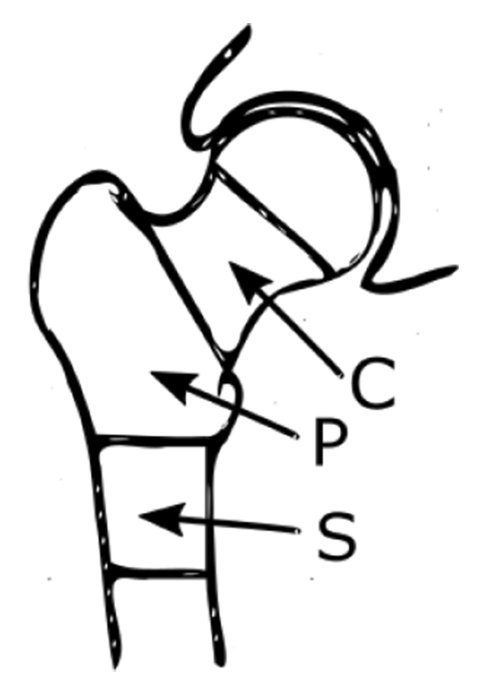

\section{C: Femoral neck fracture \\ P: Pertrochanteric fracture \\ S: Subtrochanteric fracture}

Figure I Types of collum femoris fractures included in the multidisciplinary hip fracture registry. 
delay (24 and 36 hours), mobilization within 24 hours, assessment of nutrition status, basic mobility score preand postoperative, rehabilitation program, osteoporosisand fall prophylaxis. The process performance measures reflect key recommendations for healthcare processes, which are expected to be associated with patient outcome. The staff members classify the patient as eligible or noneligible for the process performance measures according to detailed data definitions. Subsequently, the staff members register fulfillment or non-fulfillment of the measure. Missing registration of fulfillment would be coded as a nonfulfillment of the process performance measure.

Currently, three outcome measures are available: Survival within 30 days, readmission within 30 days after discharge and reoperation within two years. Due to the individual-level record linkage to other public registries and the civil registry number in Denmark, complete follow-up for every patient is possible for the outcome measures. Information on survival is obtained from the Civil Registration System, which has daily updated electronic records of all changes in vital status for the entire Danish population since $1968 .{ }^{18}$ Readmission is defined as an acute first time readmission to any hospital 24 hours after discharge from hip fracture with a length of stay of minimum 24 hours. Reoperation is defined in four different ways depending on fracture type and surgery type. Information on readmission and reoperation is obtained from the Danish National Patient Registry, which is a nationwide administrative registry that covers all admissions and discharges from Danish somatic hospitals since 1977. Since 1994, up to 20 diagnoses for every discharge are coded according to the Danish version of the International Classification of Diseases tenth edition. ${ }^{20}$

The core of the quality of care indicators has been quite consistent in the first ten years. However, demands for fulfillment of some process performance measures have increased over time and data definitions were adjusted. For instance, a timestamp for mobilization within 24 hours was implemented in 2015. Before 2015, the clinicians reported whether the patient was mobilized within 24 hours with the answer yes or no. After 2015, clinicians have reported the exact time for first mobilization. Likewise, in 2013, fulfillment of the measure basic mobility assessment presupposes that the CAS score value also was reported. Moreover, new quality indicators have been added by the steering committee regularly. Detailed specifications for the individual indicator calculation are publicly available through the Danish Clinical Registry's website. ${ }^{16}$ A description of the measures used in the registry is presented in Table 1.

Local and regional audits on quality indicators are carried out every third month. Further, the steering committee performs an annually structured audit process and publishes an annual report followed by comments and recommendations from the audits on how to improve quality of care.

\section{Descriptive Variables}

The DMHFR has information on a number of patient- and surgery-related variables, which, based on the current evidence, appear to be well-established prognostic factors. Patient-related variables included admission age, sex, Body Mass Index (BMI) and residence. In the period from 2005 to 2010 information on alcohol intake and smoking were collected. The surgery-related variables included type of fracture, fracture displacement, and type of surgery (Table 2). In the period from 2005 to 2010, information on the American Society of Anesthesiologists' (ASA) classification score was collected. From 2010 and onward, the ASA score was replaced with the Charlson Comorbidity Index (CCI). The CCI is a method of categorizing comorbidities of patients based on ICD diagnosis codes from the Danish National Patient Registry. ${ }^{21}$ Surgical delay was included in the DMHFR as a prognostic factor in the period 2006 to 2014 and as a process performance measure since 2015 .

\section{Results \\ Patient Characteristics}

During the period 2006-2018, the DMHFR included 87,803 hip fracture patients. The number of patients varied slightly during the study period, but the average was 6,800 patients (Table 2). The majority of hip fracture patients are women with a median age of 84 years, living alone. The most frequent fracture type is a femoral neck fracture and the most frequent surgery type is internal fixation. The proportion of hip fracture patients with comorbidity has increased over time (Table 2).

\section{Quality Indicators}

\section{Process Performance Measures}

For the process performance measures, improvement over the years has been observed for most of the measures even though the demands for fulfillment of the process performance measures have increased over the period (Figure 2). An exception is the process performance measures implemented in 2015, including preoperative optimization and 


\begin{tabular}{|c|c|c|c|c|c|c|c|c|c|c|}
\hline$\frac{\infty}{2}$ & & + & + & + & + & & + & + & + & + \\
\hline$\overline{\mathrm{N}}$ & & + & + & + & + & & + & + & + & + \\
\hline$\stackrel{\circ}{\circ}$ & & + & + & + & + & & + & + & + & + \\
\hline$\frac{n}{2}$ & & $\stackrel{\varpi}{+}$ & + & + & + & & + & + & + & + \\
\hline 广̃ & + & + & & + & + & & + & + & + & \\
\hline$\frac{m}{i}$ & + & + & & + & + & & + & $\stackrel{ }{+}$ & + & \\
\hline ָั & + & + & & + & + & & + & + & + & \\
\hline$\overline{\bar{i}}$ & + & + & & + & + & & + & + & + & \\
\hline$\frac{0}{2}$ & + & + & & + & + & & + & $\stackrel{\pi}{+}$ & + & \\
\hline ذેे & + & & + & & & + & + & + & + & \\
\hline$\stackrel{\infty}{\circ}$ & + & & + & & & + & + & + & + & \\
\hline ఏે̀ & + & & + & & & + & + & + & + & \\
\hline ஃั & + & & + & & & + & & + & + & \\
\hline 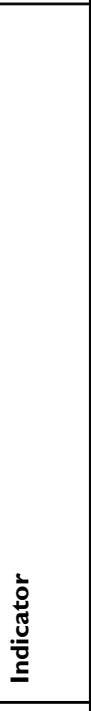 & 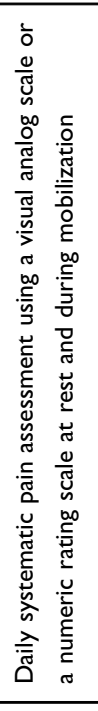 & 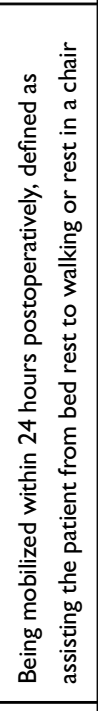 & 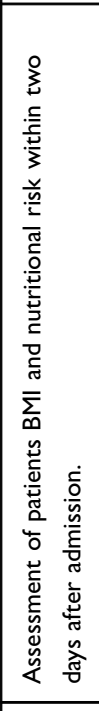 & 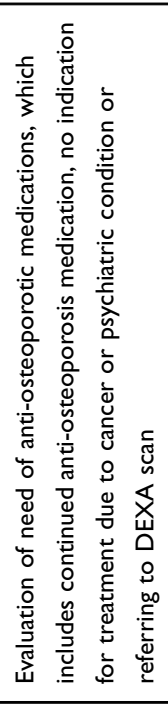 & 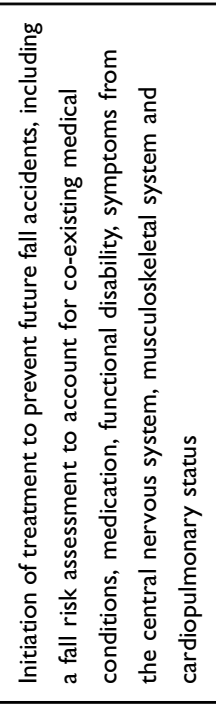 & 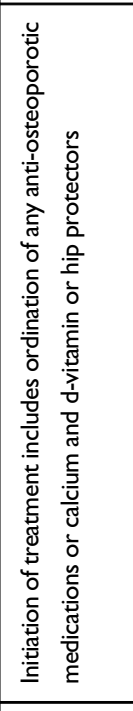 & 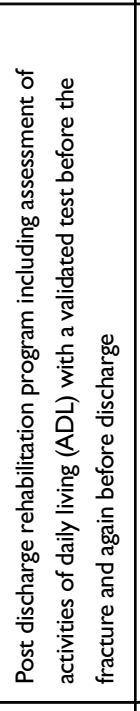 & 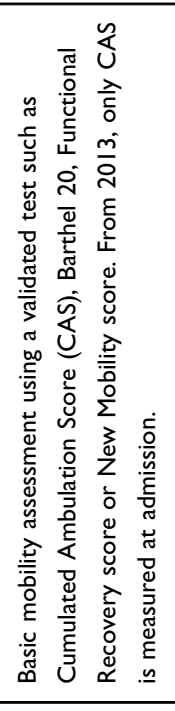 & 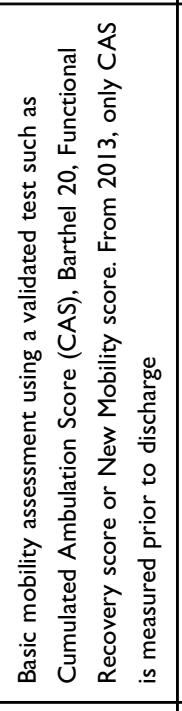 & 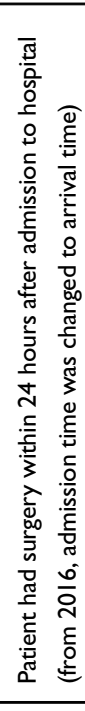 \\
\hline 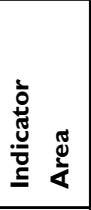 & 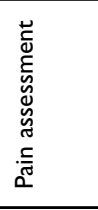 & 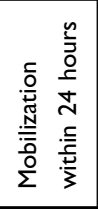 & 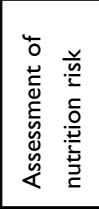 & 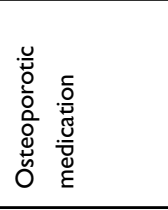 & 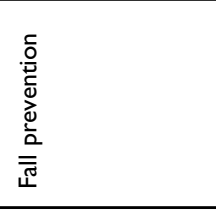 & 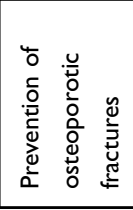 & 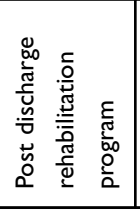 & 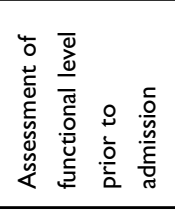 & 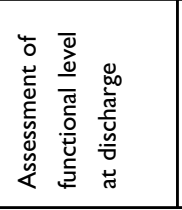 & 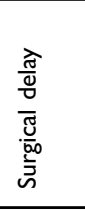 \\
\hline & 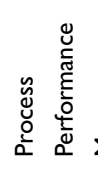 & & & & & & & & & \\
\hline
\end{tabular}




\begin{tabular}{|c|c|c|c|c|c|c|c|c|c|}
\hline+ & + & + & + & + & + & & + & + & + \\
\hline+ & + & + & + & + & + & & + & + & + \\
\hline+ & + & + & + & + & + & & + & + & + \\
\hline+ & & + & + & + & + & & + & + & \\
\hline & & + & + & + & + & & + & + & \\
\hline & & + & + & + & + & & + & + & \\
\hline & & + & + & + & + & & + & + & \\
\hline & & + & + & + & + & & + & + & \\
\hline & & + & + & + & + & & + & + & \\
\hline & & + & + & + & + & & + & + & \\
\hline & & + & + & + & + & & + & + & \\
\hline & & + & + & + & + & & + & + & \\
\hline & & + & + & + & + & & + & + & \\
\hline 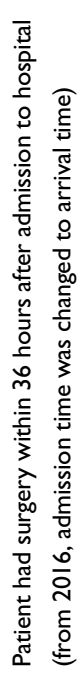 & 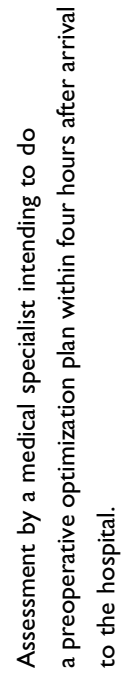 & 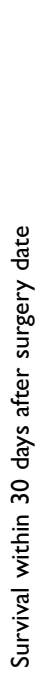 & 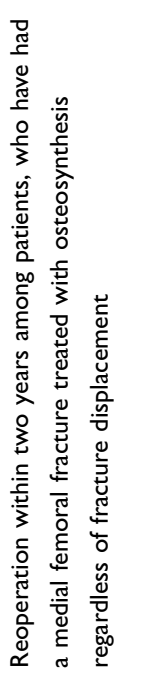 & 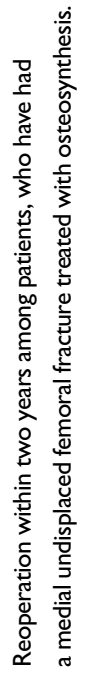 & 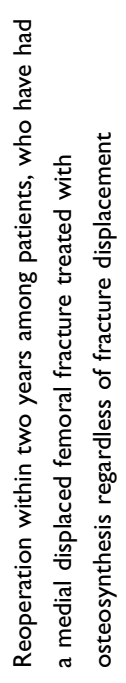 & 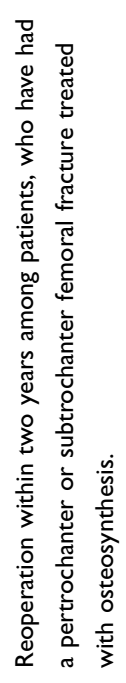 & 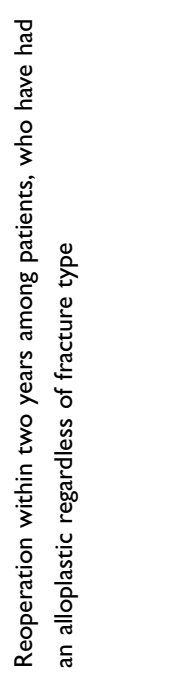 & 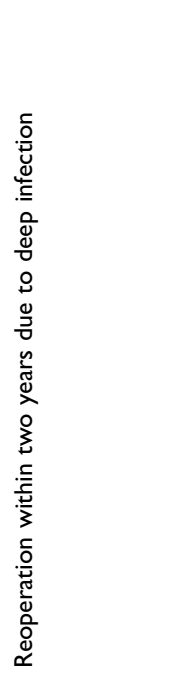 & 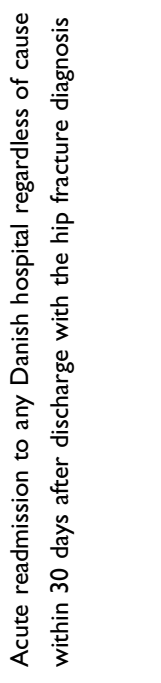 \\
\hline & 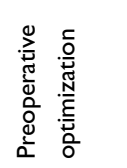 & 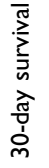 & 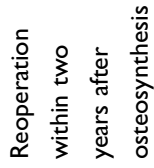 & & & & 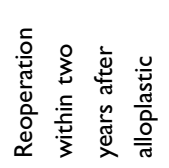 & 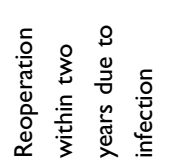 & 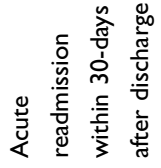 \\
\hline & & \multicolumn{8}{|c|}{ 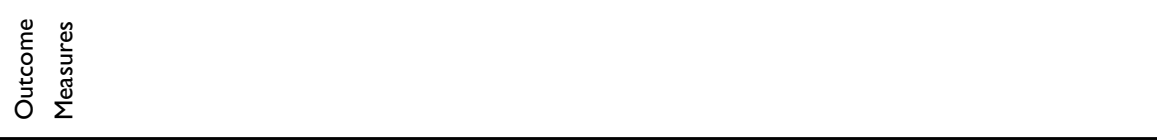 } \\
\hline
\end{tabular}




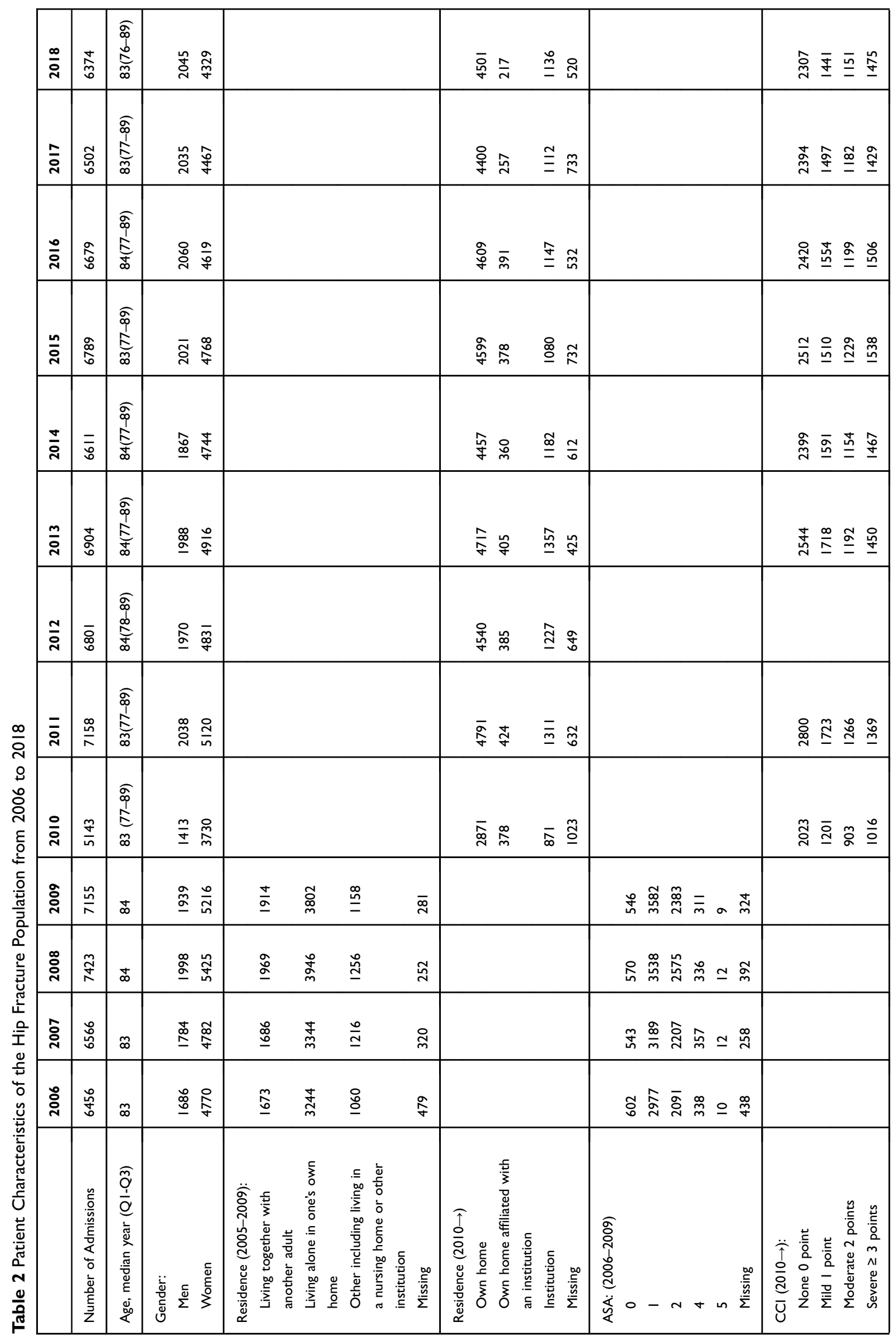




\begin{tabular}{|c|c|c|c|c|c|c|c|}
\hline 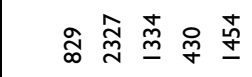 & 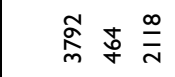 & 命突 & 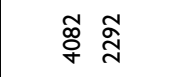 & & & & \\
\hline 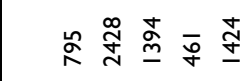 & 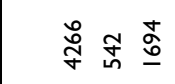 & 离品 & $\stackrel{゚}{\frac{\sigma}{\sigma}} \underset{\sim}{\sim}$ & & & & \\
\hline 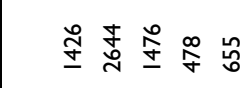 & 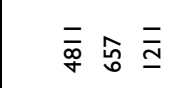 & 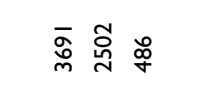 & 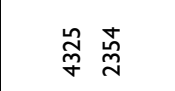 & & & & \\
\hline 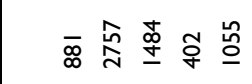 & 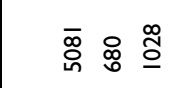 & 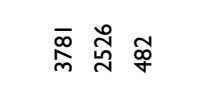 & 学 & & & & \\
\hline 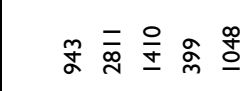 & 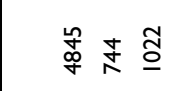 & 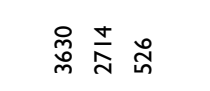 & 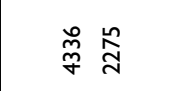 & & & & 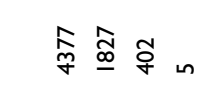 \\
\hline 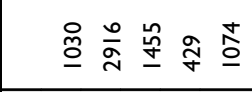 & $\underset{\text { กิ }}{\infty} \underset{\infty}{\mathbb{N}} \underset{\infty}{+}$ & 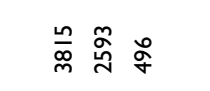 & 紊早 & & & & 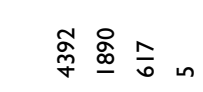 \\
\hline 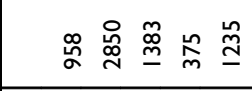 & 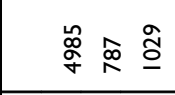 & 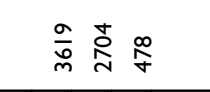 & 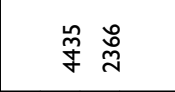 & & & & 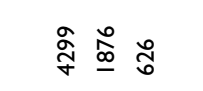 \\
\hline 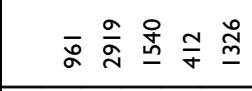 & 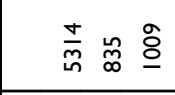 & గ్ల & 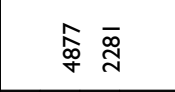 & & & & 总 $\underset{\sim}{\infty} \underset{\sim}{\infty} \stackrel{m}{\infty} n$ \\
\hline 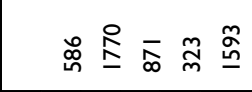 & $\frac{\tilde{g}}{m}$ 守 & 孞 & 惫 & & & & 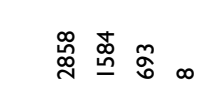 \\
\hline & 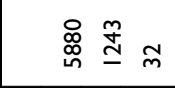 & 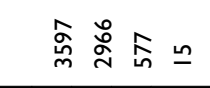 & 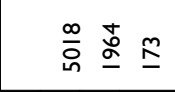 & 商 & $\stackrel{\sim}{\pi} \quad \underline{0}$ & 芯家器 & 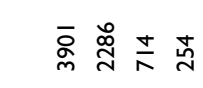 \\
\hline & 志 空 & $\stackrel{\infty}{\infty} \underset{m}{\stackrel{n}{\circ}} \overline{n_{n}}=$ & 总 串 & în & $\underset{N}{\stackrel{N}{=}}$ & 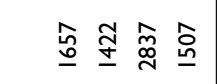 & 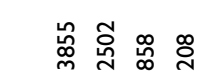 \\
\hline & $\stackrel{\substack{n \\
\hat{n}}}{\stackrel{g}{=}} q$ & 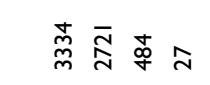 & 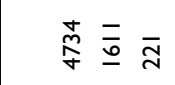 & $\overline{\grave{o}}$ & 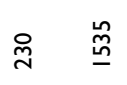 & 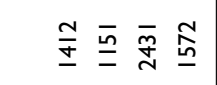 & 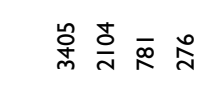 \\
\hline & 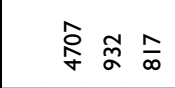 & 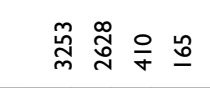 & 合尽孯 & 等 & $\begin{array}{ll}\stackrel{\infty}{\infty} & \stackrel{\mathscr{\infty}}{\infty} \\
\underline{0}\end{array}$ & 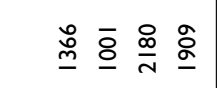 & 命余令 \\
\hline 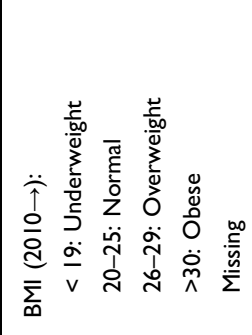 & 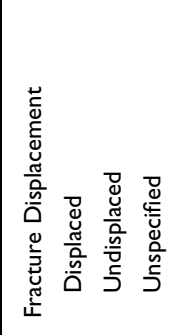 & 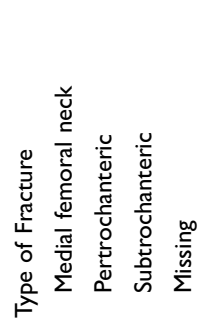 & 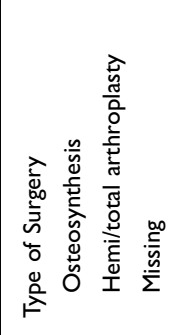 & 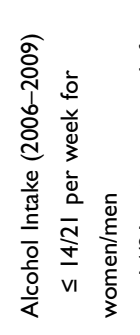 & 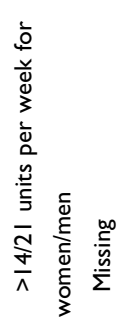 & 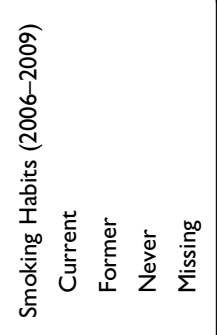 & 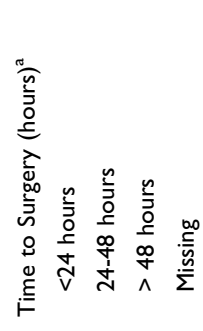 \\
\hline
\end{tabular}




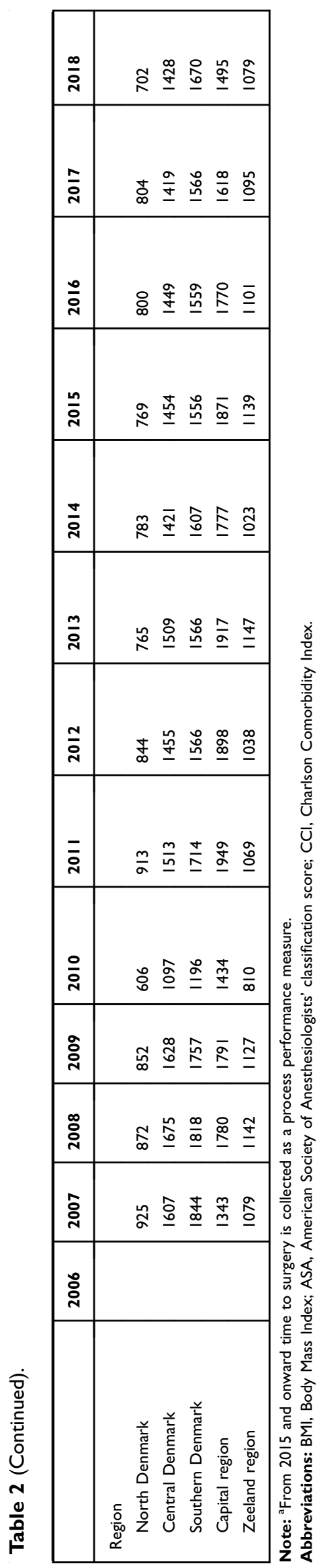

surgery within 24 and 36 hours respectively, where the fulfillment has not improved over the three years. A decrease in fulfillment of some of the process performance measures such as mobilization within 24 hours in 2015 and basic mobility assessment in 2013 may be related to change in data definitions (Figure 2).

\section{Outcome Measures 30-Day Mortality, Readmission and Reoperation}

The overall 30-day survival varied from $90 \%$ to $88 \%$ during 2006-2018. The proportion of patients that were re-operated within two years has decreased over the study period, from $27 \%$ to $13 \%$ among patients with a displaced medial femoral neck fracture, and from $15 \%$ to $9 \%$ among patients with un-displaced medial fracture (Figure 3). Reoperations among patients treated with an arthroplasty were highest in the period from 2008 to 2011 with around $13 \%$, and have decreased to $9 \%$ subsequently. Almost $1820 \%$ of patients were readmitted within 30 days during 2015 to 2018 .

\section{Examples of Research}

The DMHFR data are accessible for research after application to the Danish Clinical Registries. ${ }^{16}$ Published papers are based on the DMHFR data linked to other Danish health registries, including the Danish National Patient Registry, the Civil Registration System, the Danish Transfusion Database, the Danish Prescription Database, the Laboratory Information System, and the Integrated Database for Labour Marked Research. The studies have focused on variation in the outcome measures among patient types and hospitals. ${ }^{22-24}$ An association between fulfillment of the process performance measures and better survival and lower readmission rate has been found ${ }^{25-28}$ as well as an association between orthogeriatric specialization or patient volume and outcome. ${ }^{29-31}$ In addition, associations between transfusion practice and outcome, ${ }^{32,33}$ association between surgery delay and post-operative complications, ${ }^{34,35}$ associations between some commonly used medication preoperatively, including antidepressants, anticoagulants and anti-inflammatory drugs and post-operative complications $^{36-40}$ have been observed. Moreover, studies have found that about $15 \%$ of hip fracture patients develop an acute kidney injury within 5 days of surgery ${ }^{41,42}$ and about $15 \%$ of patients sustain postoperative infections within 30 days of surgery, ${ }^{43,44}$ 


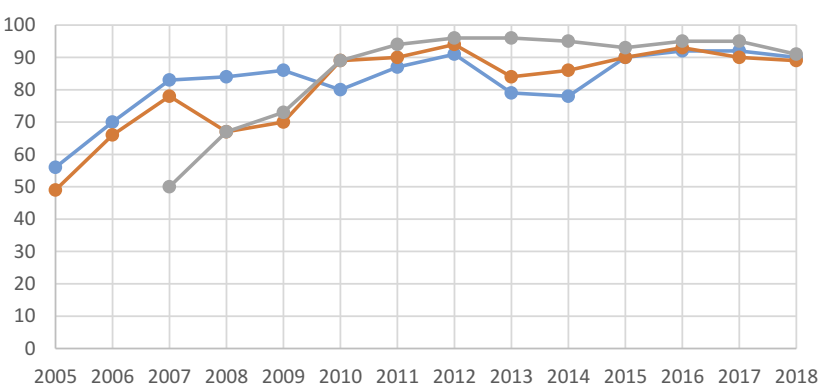

$\longrightarrow$ ADL/CAS_Admission $\rightarrow$ ADL/CAS_Discharge $\multimap$ Rehabilitation

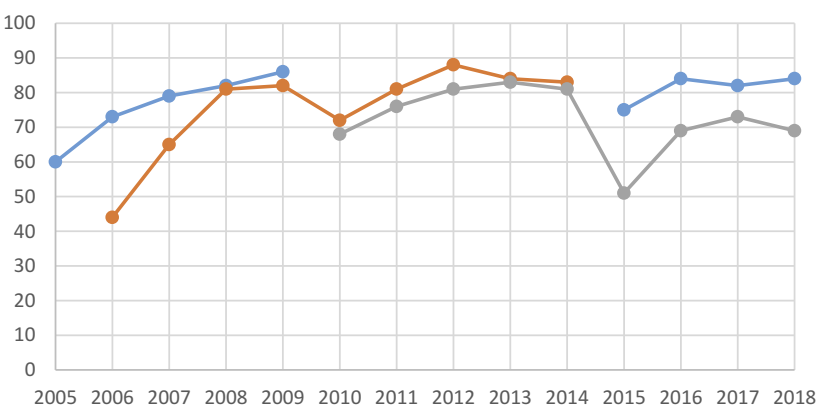

$\longrightarrow$ Nutrition $\longrightarrow$ Pain assessment $\longrightarrow$ Mobilization_24h

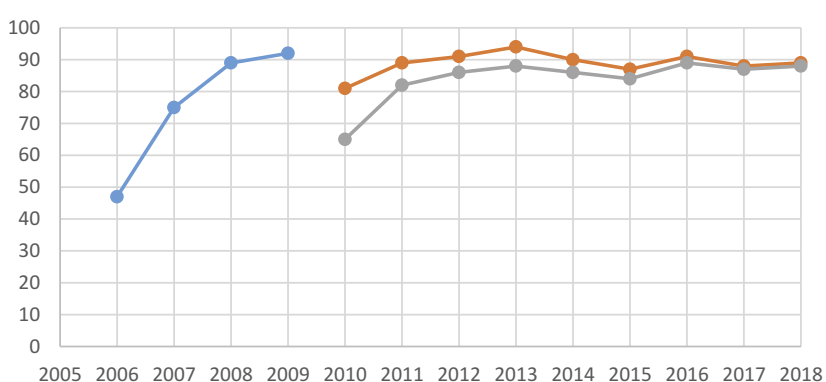

$\multimap$ Fracture_prophylaxis $\longrightarrow$ Osteoporosis_prophylaxis $\longrightarrow$ Fall_prophylaxis

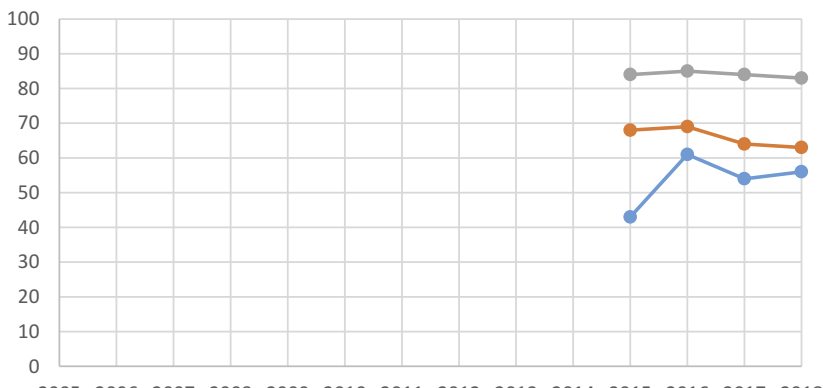

$\longrightarrow$-Pre_optimization $\rightarrow$ Op_delay_24h $\rightarrow$ Op_delay_36h

Figure 2 Proportion of patients in percent that have fulfilled the individual process performance measures over time.

complications which are strongly associated with 30 days mortality. Besides, hip fracture patients were at increased excess risk of venous thromboembolism, myocardial infarction and stroke up to one year following fracture. ${ }^{45,46}$

\section{Discussion}

The DMHFR is a nationwide registry established in 2003 with the aim of monitoring and improving hip fracture treatment and care in Denmark. The hip fracture population in Denmark is characterized by a median age of 84

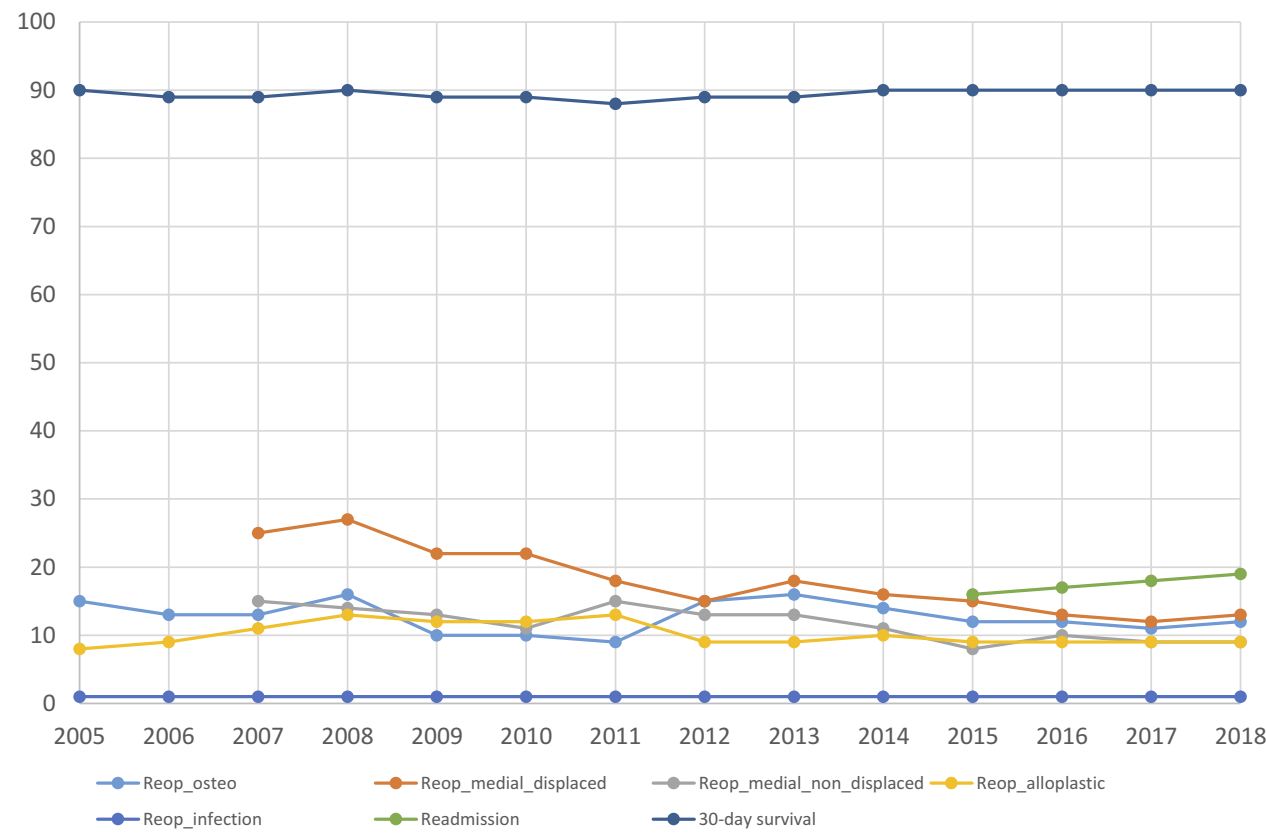

Figure 3 Proportion of patients with 30-day survival and readmission and reoperation within two years over time. 
years and a high completeness of reporting. The quality of treatment and care reflected by receiving guideline recommended recommendations has increased over the years. The 30-day survival has improved from $88 \%$ to $90 \%$ in the period. The proportion of reoperations has decreased especially among patients with medial femoral neck fracture. In contrast, the risk for acute readmission has increased from $16 \%$ to $20 \%$ in the last three years.

\section{Comparison with Other Hip Fracture Registries}

Continuous monitoring of the quality of healthcare has been implemented in many European countries ${ }^{14}$ including Finland (PERFormance, Effectiveness and Cost of Treatment episodes), ${ }^{47}$ Sweden (Rikshöft), ${ }^{48}$ the United Kingdom (UK) (the National Hip Fracture Database), ${ }^{13,49}$ Scotland (the Scottish Hip Fracture Audit), ${ }^{50}$ Italy (Regional Outcome Evaluation Program in the Lazio region ${ }^{51}$ and Gruppo Italiano di Ortogeriatria), ${ }^{52}$ Norway (The Norwegian Hip Fracture Registry), ${ }^{53}$ Spain (National Hip Fracture Registry), ${ }^{54}$ Ireland (Irish Hip Fracture database), ${ }^{55}$ Nederlands (Dutch National Hip Fracture Audit), ${ }^{56}$ Germany (Alterstrauma register),${ }^{57}$ and Denmark (DMHFR). However, the majority of these registries ${ }^{47,50,51,53-55}$ mainly monitor outcome performance measures, eg, mortality and readmissions at the hospital level, and lack continuous monitoring of process performance measures. Exceptions are the DMHFR, Rikshö $\mathrm{ft}^{48}$ and the National Hip Fracture Database in the UK, ${ }^{13,49}$ which contain valuable information on process performance measures according to clinical guideline recommendations. The measures monitored in the National Hip Fracture Database in the UK are comparable to the process performance measures as they monitor mobilization on the first postoperative day, bone-protection medication and geriatric assessment. The fulfillment of the process performance measures is generally higher in the UK compared to Denmark, eg, $69 \%$ were mobilized on the first operative day in Denmark whereas $79 \%$ were mobilized in the UK. Likewise is the survival lower in Denmark compared to the UK and Sweden, as they report an overall 30-day mortality rate below $8 \%$ compared to $10 \%$ in Denmark. ${ }^{58,59}$ The lower mortality may be explained by different inclusion criteria in the hip fracture registries, eg, different inclusion ages, which may impact the risk of mortality. For instance, the Scottish Hip Fracture Audit, the Swedish Rikshöft registry and the Finish PERFECT registry include hip fracture patients from 50 years, whereas the Danish Multidisciplinary hip fracture registry includes patients from 65 years.

\section{Perspective}

The efforts are on-going to improve the fulfillment of the process performance measures and thereby improve the hip fracture patients' survival. The completeness of registered variables for each patient is high, however an indepth validation of the data in the DMHFR with focus on completeness and positive predictive value of the registered data needs to be done. The length of hospital stay has decreased, which includes earlier discharge to care in the community settings or at home with support from home care or mobile rehabilitation units. The hip fracture registries therefore need data from the community setting including primary healthcare services to improve our knowledge on the full clinical pathway of hip fracture patients and it impact on the patient outcome.

\section{Administrative Issues and Funding}

The clinicians reporting to the registry have no economic incentives, but there is an increasing interest from politicians, hospital boards of directors and patient organizations regarding fulfillment of the quality indicators. DMHFR is funded by the Danish Regions and receives epidemiological, statistical and administrative support from the Danish Clinical Registries (RKKP), which has a budget of 9.9 million USD to operate 80 databases in Denmark. DMHFR receives 16,000 USD yearly for holding audits, multidisciplinary seminars and revising data definitions. The DMHFR is approved as a national clinical quality database by the National Board of Health and the Data Protection Agency. Permission to access the data from the DMHFR has been granted by the Data Protection Agency and the steering Committee following an application to the Data Protection Agency and RKKP.

\section{Conclusion}

The DMHFR plays an important key role in monitoring and improving hip fracture care in Denmark due to prospectively collected high-validity data. Furthermore, the DMHFR has been linked to a wide range of other national registries in order to answer a number of relevant clinical questions regarding the treatment and outcome of hip fracture patients. Thus, the DMHFR is a valuable tool for both quality improvement and epidemiological research.

\section{Ethics}

The study was approved by the Danish Data Protection Agency (journal number 2012-41-1274) and carried out in accordance with the principle of the Declaration of Helsinki. 
The requirement for consent was waived as registry-based studies do not require formal ethical approval according to the Danish law. Patient data was kept confidential.

\section{Acknowledgments}

We thank the staff at the hospital departments involved in treatment of patients with hip fracture for their continuous effort and contribution in acquiring the data in the DMHFR. Also, we thank the steering Committee of the DMHFR for their ongoing effort with development, interpretation and evaluation of quality indicators and thereby quality improvement.

\section{Funding}

This work was supported by the Health Research Fund of Central Denmark Region (Pia Kjær Kristensen). The financial sponsors did not play a role in the design, execution, analysis, interpretation of data, or writing of the study.

\section{Disclosure}

Pia Kristensen reports grants from Health Research Fund of Central Denmark Region, during the conduct of the study. The authors report no other conflicts of interest in this work.

\section{References}

1. Kanis JA, Oden A, McCloskey EV, et al. A systematic review of hip fracture incidence and probability of fracture worldwide. Osteoporos Int. 2012;23(9):2239-2256. doi:10.1007/s00198-012-1964-3

2. Singer A, Exuzides A, Spangler L, et al. Burden of illness for osteoporotic fractures compared with other serious diseases among postmenopausal women in the United States. Mayo Clin Proc. 2015;90 (1):53-62. doi:10.1016/j.mayocp.2014.09.011

3. Roche JJ, Wenn RT, Sahota O, Moran CG. Effect of comorbidities and postoperative complications on mortality after hip fracture in elderly people: prospective observational cohort study. BMJ. 2005;331 (7529):1374. doi:10.1136/bmj.38643.663843.55

4. Cummings SR, Melton LJ. Epidemiology and outcomes of osteoporotic fractures. Lancet. 2002;359(9319):1761-1767. doi:10.1016/S01406736(02)08657-9

5. Cauley JA. Public health impact of osteoporosis. J Gerontol a Biol Sci Med Sci. 2013;68(10):1243-1251. doi:10.1093/gerona/glt093

6. Katsoulis M, Benetou V, Karapetyan T, et al. Excess mortality after hip fracture in elderly persons from Europe and the USA: the CHANCES project. J Intern Med. 2017;281(3):300-310. doi:10.1111/joim.12586

7. Brauer CA, Coca-Perraillon M, Cutler DM, Rosen AB. Incidence and mortality of hip fractures in the United States. JAMA. 2009;302 (14):1573-1579. doi:10.1001/jama.2009.1462

8. Tosteson AN, Burge RT, Marshall DA, Lindsay R. Therapies for treatment of osteoporosis in US women: cost-effectiveness and budget impact considerations. Am J Manag Care. 2008;14(9):605-615.
9. Burge R, Dawson-Hughes B, Solomon DH, Wong JB, King A, Tosteson A. Incidence and economic burden of osteoporosis-related fractures in the United States, 2005-2025. J Bone Miner Res. 2007;22 (3):465-475. doi:10.1359/jbmr.061113

10. Cauley JA, Chalhoub D, Kassem AM, Fuleihan G-H. Geographic and ethnic disparities in osteoporotic fractures. Nat Rev Endocrinol. 2014;10(6):338-351.

11. Fisher ES, Wennberg DE, Stukel TA, Gottlieb DJ, Lucas FL, Pinder EL. The implications of regional variations in medicare spending. Part 2: health outcomes and satisfaction with care. Ann Intern Med. 2003;138 (4):288-298. doi:10.7326/0003-4819-138-4-200302180-00007

12. Thygesen LC, Christiansen T, Garcia-Armesto S et al. Potentially avoidable hospitalizations in five European countries in 2009 and time trends from 2002 to 2009 based on administrative data. European Journal of Public Health. 2015;25(suppl_1):35-43.

13. Patel NK, Sarraf KM, Joseph S, Lee C, Middleton FR. Implementing the national hip fracture database: an audit of care. Injury. 2013;44 (12):1934-1939. doi:10.1016/j.injury.2013.04.012

14. Saez-Lopez P, Branas F, Sanchez-Hernandez N, Alonso-Garcia N, Gonzalez-Montalvo JI. Hip fracture registries: utility, description, and comparison. Osteoporos Int. 2017;28(4):1157-1166. doi:10.1007/s00198016-3834-x

15. Mainz J, Krog BR, Bjornshave B, Bartels P. Nationwide continuous quality improvement using clinical indicators: the Danish National Indicator Project. Int J Qual Health Care. 2004;16(Suppl 1):i45-I50. doi:10.1093/intqhe/mzh031

16. Regionernes Kliniske Kvalitets Program. De kliniske kvalitetsdatabaser. 2015. Available from: http://www.rkkp.dk/. Accessed January 10, 2019.

17. Schmidt M, Schmidt SAJ, Adelborg K, et al. The Danish health care system and epidemiological research: from health care contacts to database records. Clin Epidemiol. 2019;11:563. doi:10.2147/CLEP.S179083

18. Schmidt M, Pedersen L, Sorensen HT. The Danish Civil Registration System as a tool in epidemiology. Eur J Epidemiol. 2014;29 (8):541-549. doi:10.1007/s10654-014-9930-3

19. Ministry D. The Danish health act legislation number 546 from 24 June 2005. Danish legislation number 546. Vol 546.

20. Schmidt M, Schmidt SA, Sandegaard JL, Ehrenstein V, Pedersen L, Sorensen HT. The Danish National Patient Registry: a review of content, data quality, and research potential. Clin Epidemiol. 2015;7:449-490. doi:10.2147/CLEP.S91125

21. Kirkland LL, Kashiwagi DT, Burton MC, Cha S, Varkey P. The Charlson Comorbidity Index Score as a predictor of 30-day mortality after hip fracture surgery. Am J Med Qual. 2011;26(6):461-467. doi:10.1177/1062860611402188

22. Kristensen PK, Merlo J, Ghith N, Leckie G, Johnsen SP. Hospital differences in mortality rates after hip fracture surgery in Denmark. Clin Epidemiol. 2019;11:605. doi:10.2147/CLEP.S213898

23. Kristensen PK, Johnsen SP, Mor A, Thillemann TM, Pedersen AB. Is the higher mortality among men with hip fracture explained by sex-related differences in quality of in-hospital care? A population-based cohort study. Age Ageing. 2017;46(2):193-199. doi:10.1093/ageing/afw225

24. Kristensen PK, Thillemann TM, Pedersen AB, Soballe K, Johnsen SP. Socioeconomic inequality in clinical outcome among hip fracture patients: a nationwide cohort study. Osteoporos Int. 2017;28(4):1233-1243. doi:10.1007/s00198-016-3853-7

25. Kristensen PK, Thillemann TM, Soballe K, Johnsen SP. Are process performance measures associated with clinical outcomes among patients with hip fractures? A population-based cohort study. Int J Qual Health Care. 2016;28(6):698-708. doi:10.1093/intqhc/mzw093

26. Nielsen KA, Jensen NC, Jensen CM, et al. Quality of care and 30 day mortality among patients with hip fractures: a nationwide cohort study. BMC Health Serv Res. 2009;9:186-6963-6969-6186. doi:10.11 86/1472-6963-9-186 
27. Kristensen PK, Sogaard R, Thillemann TM, Soballe K, Johnsen SP. High quality of care did not imply increased hospital spendingnationwide cohort study among hip fracture patients. Int $J$ Qual Health Care. 2019. doi:10.1093/intqhc/mzy243

28. Kristensen MT, Ozturk B, Rock ND, Ingeman A, Palm H, Pedersen AB. Regaining pre-fracture basic mobility status after hip fracture and association with post-discharge mortality and readmission-a nationwide register study in Denmark. Age Ageing. 2019;48(2):278-284. doi:10.1093/ageing/afy185

29. Kristensen PK, Thillemann TM, Johnsen SP. Is bigger always better? A nationwide study of hip fracture unit volume, 30-day mortality, quality of in-hospital care, and length of hospital stay. Med Care. 2014;52(12):1023-1029. doi:10.1097/MLR.0000000000000234

30. Kristensen PK, Thillemann TM, Soballe K, Johnsen SP. Can improved quality of care explain the success of orthogeriatric units? A population-based cohort study. Age Ageing. 2015;45:66-71.

31. Kristiansen NS, Kristensen PK, Norgard BM, Mainz J, Johnsen SP. Offhours admission and quality of hip fracture care: a nationwide cohort study of performance measures and 30-day mortality. Int J Qual Health Care. 2016;28(3):324-331. doi:10.1093/intqhe/mzw037

32. Viberg B, Gundtoft PH, Schonnemann J, et al. Introduction of national guidelines for restrictive blood transfusion threshold for hip fracture patients-a consecutive cohort study based on complete follow-up in national databases. J Orthop Surg Res. 2018;13(1):116. doi:10.1186/s13018-018-0828-8

33. Pedersen AB, Cronin Fenton D, Norgaard M, Kristensen NR, Kuno Moller B, Erikstrup C. Body mass index, risk of allogeneic red blood cell transfusion, and mortality in elderly patients undergoing hip fracture surgery. Osteoporos Int. 2016;27(9):2765-2775. doi:10.1007/s00198-016-3594-7

34. Glassou EN, Kjorholt KK, Hansen TB, Pedersen AB. Delay in surgery, risk of hospital-treated infections and the prognostic impact of comorbidity in hip fracture patients. A Danish nationwide cohort study, 2005-2016. Clin Epidemiol. 2019;11:383-395. doi:10.2147/ CLEP.S200454

35. Ozturk B, Johnsen SP, Rock ND, Pedersen L, Pedersen AB. Impact of comorbidity on the association between surgery delay and mortality in hip fracture patients: a Danish nationwide cohort study. Injury. 2019;50(2):424-431. doi:10.1016/j.injury.2018.12.032

36. Bruun SB, Petersen I, Kristensen NR, Cronin-Fenton D, Pedersen AB. Selective serotonin reuptake inhibitor use and mortality, postoperative complications, and quality of care in hip fracture patients: a Danish nationwide cohort study. Clin Epidemiol. 2018;10:1053-1071. doi:10.2147/CLEP.S166309

37. Daugaard C, Pedersen AB, Kristensen NR, Johnsen SP. Preoperative antithrombotic therapy and risk of blood transfusion and mortality following hip fracture surgery: a Danish nationwide cohort study. Osteoporos Int. 2019;30(3):583-591. doi:10.1007/s00198-018-4786-0

38. Glassou EN, Kristensen N, Moller BK, Erikstrup C, Hansen TB, Pedersen AB. Impact of preadmission anti-inflammatory drug use on the risk of RBC transfusion in elderly hip fracture patients: a Danish nationwide cohort study, 2005-2016. Transfusion. 2019;59 (3):935-944. doi:10.1111/trf.2019.59.issue-3

39. Bruun SB, Petersen I, Kristensen NR, Cronin-Fenton D, Pedersen AB. Selective serotonin reuptake inhibitor use in hip fracture patients: a Danish nationwide prevalence study. Acta Orthop. 2019;90(1):33-39. doi:10.1080/17453674.2018.1543842

40. Simoni AH, Nikolajsen L, Olesen AE, Christiansen CF, Pedersen AB. Opioid use after hip fracture surgery: a Danish nationwide cohort study from 2005 to 2015. Eur J Pain. 2019;23 (7):1309-1317. doi:10.1002/ejp.1392
41. Pedersen AB, Christiansen CF, Gammelager H, Kahlert J, Sorensen HT. Risk of acute renal failure and mortality after surgery for a fracture of the hip: a population-based cohort study. Bone Joint J. 2016;98-b(8):1112-1118. doi:10.1302/0301-620X.98B8.37497

42. Pedersen AB, Gammelager H, Kahlert J, Sorensen HT, Christiansen CF. Impact of body mass index on risk of acute kidney injury and mortality in elderly patients undergoing hip fracture surgery. Osteoporos Int. 2017;28(3):1087-1097. doi:10. 1007/s00198-016-3836-8

43. Kjorholt KE, Kristensen NR, Prieto-Alhambra D, Johnsen SP, Pedersen $\mathrm{AB}$. Increased risk of mortality after postoperative infection in hip fracture patients. Bone. 2019;127:563-570. doi:10.1016/j.bone.20 19.07.023

44. Kjorholt KE, Johnsen SP, Kristensen NR, Prieto-Alhambra D, Pedersen AB. Increasing risk of hospital-treated infections and community-based antibiotic use after hip fracture surgery: a nationwide study 2005-2016. J Bone Miner Res. 2019;34(3):437-446. doi:10.1002/ jbmr.3620

45. Pedersen AB, Ehrenstein V, Szepligeti SK, Sorensen HT. Excess risk of venous thromboembolism in hip fracture patients and the prognostic impact of comorbidity. Osteoporos Int. 2017;28 (12):3421-3430. doi:10.1007/s00198-017-4213-y

46. Pedersen AB, Ehrenstein V, Szepligeti SK, Sorensen HT. Hip fracture, comorbidity, and the risk of myocardial infarction and stroke: a Danish nationwide cohort study, 1995-2015. J Bone Miner Res. 2017;32(12):2339-2346. doi:10.1002/jbmr.v32.12

47. Sund R, Juntunen M, Luthje P, Huusko T, Hakkinen U. Monitoring the performance of hip fracture treatment in Finland. Ann Med. 2011;43(Suppl 1):S39-S46. doi:10.3109/07853890.2011.586 360

48. Thorngren KG. National registration of hip fractures. Acta Orthop. 2008;79(5):580-582. doi:10.1080/17453670810016579

49. Hawkes D, Baxter J, Bailey C, et al. Improving the care of patients with a hip fracture: a quality improvement report. BMJ Qual Saf. 2015;24(8):532-538. doi:10.1136/bmjqs-2014-003700

50. Farrow L, Hall A, Wood AD, et al. Quality of care in hip fracture patients: the relationship between adherence to national standards and improved outcomes. J Bone Joint Surg Am. 2018;100(9):751-757. doi:10.2106/JBJS.17.00884

51. Renzi C, Sorge C, Fusco D, Agabiti N, Davoli M, Perucci CA. Reporting of quality indicators and improvement in hospital performance: the P.Re.Val.E. regional outcome evaluation program. Health Serv Res. 2012;47(5):1880-1901. doi:10.1111/hesr.2012.47. issue-5

52. Zurlo Amedeo BG. Orthogeriatrics in Italy: the Gruppo Italiano di Ortogeriatria (GIOG) audit on hip fractures in the elderly. Geriatr Care. 2018;4(2):33-35.

53. Gjertsen JE, Dybvik E, Furnes O, et al. Improved outcome after hip fracture surgery in Norway. Acta Orthop. 2017;88(5):505-511. doi:10.1080/17453674.2017.1344456

54. Ojeda-Thies C, Saez-Lopez P, Currie CT, et al. Spanish National Hip Fracture Registry (RNFC): analysis of its first annual report and international comparison with other established registries. Osteoporos Int. 2019;30(6):1243-1254. doi:10.1007/s00198-01904939-2

55. Hughes AJ, Hennessy O, Brennan L, Rana A, Murphy CG. How accurate is the data provided to the Irish hip fracture database? Ir J Med Sci. 2019;188(1):13-18. doi:10.1007/s11845-018-1810-5

56. Voeten SC, Arends AJ, Wouters MWJM, et al. The Dutch Hip Fracture Audit: evaluation of the quality of multidisciplinary hip fracture care in the Netherlands. Arch Osteoporos. 2019;14(1):28. doi:10.1007/s11657-019-0576-3 
57. der Unfallchirurgie AA, AA DGU. Das AltersTraumaRegister DGU $^{\circledR}$-aktueller Stand, Methodik und Publikationsrichtlinie. Unfallchirurg. 2019;122:1-3.

58. Mattisson L, Bojan A, Enocson A. Epidemiology, treatment and mortality of trochanteric and subtrochanteric hip fractures: data from the Swedish fracture register. BMC Musculoskelet Disord. 2018. doi:10.1186/s12891-018-2276-3
59. Sayers A, Whitehouse MR, Berstock JR, Harding KA, Kelly MB, Chesser TJ. The association between the day of the week of milestones in the care pathway of patients with hip fracture and 30-day mortality: findings from a prospective national registry - The National Hip Fracture Database of England and Wales. BMC Med. 2017. doi:10.1186/s12916017-0825-5

\section{Publish your work in this journal}

Clinical Epidemiology is an international, peer-reviewed, open access, online journal focusing on disease and drug epidemiology, identification of risk factors and screening procedures to develop optimal preventative initiatives and programs. Specific topics include: diagnosis, prognosis, treatment, screening, prevention, risk factor modification,

Submit your manuscript here: https://www.dovepress.com/clinical-epidemiology-journal systematic reviews, risk \& safety of medical interventions, epidemiology \& biostatistical methods, and evaluation of guidelines, translational medicine, health policies \& economic evaluations. The manuscript management system is completely online and includes a very quick and fair peer-review system, which is all easy to use. 